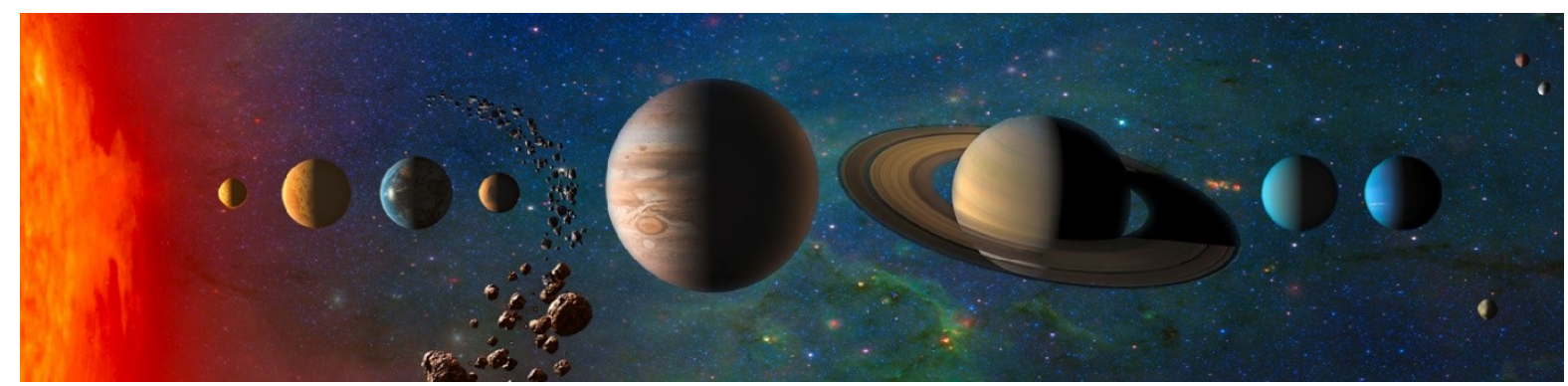

\title{
Astrobiological Group of Social Issues for the Planetary Science and Astrobiology Decadal Survey 2023-2032
}

\author{
Author \\ FLORA ARDENGHI DUTRA \\ floradutra@gmail.com \\ Federal University of Rio Grande do Sul \\ Graduate Department in Social Anthropology \\ Co-author \\ ALEXEY DODSWORTH MAGNAVITA DE CARVALHO \\ alexey.dodsworth@gmail.com \\ University of São Paulo \\ Doctor of Philosophy
}

Submitted to:

Planetary Science and Astrobiology Decadal Survey 2023-2032

The National Academy of Sciences, Engineering and Medicine

Flora A. Dutra

+55 (55) 98445-7911

floradutra.com

Alexey D. M. de Carvalho

+55 (55) 98413-5035 


\section{Introduction - A chapter in the history of Astrobiology for the Humanities}

Over the last few decades, the interdisciplinary approach of Astrobiology has drawn worldwide attention. Astrobiology is devoted not only to the search for life in extraterrestrial contexts, but also focuses on understanding the impact of cosmic factors on our planet. Given this scope, Astrobiology revives our ancient awe and wonder in the face of the fundamental questions that guide human existence. Yet is from outside the Natural Sciences, from social scientists and scholars of diverse areas of the Humanities, that come the discussions that have the capacity to make Astrobiology truly interdisciplinary, and a matter of attention within the context of diverse fields of knowledge. The moral and ethical principles that provide the foundations of the astrobiological field in the first Roadmaps (1998, 2003, 2008) deal directly with the interdisciplinary content and coordination of several scientific disciplines and programs. They promote concerns with Defence and Planetary Protection, linked to the ethical issues associated with the exploration of outer space and recognizing a broad interest of global society in the social implications of the search for life in the universe, and for other worlds beyond Earth. It is in this urgent and emerging context within Astrobiology that we propose the Astrobiological Group of Social Issues for the Planetary Science and Astrobiology Decadal Survey 2023-2032. This paper presents our claims, actions, and suggestions for work over the upcoming years that is able to connect the Humanities and Astrobiology within a social context-oriented toward public education and the dissemination of science.

Nonetheless, the strategic 'Reports' (2015, 2017, 2018, 2019) that follow the publication of the Roadmaps give very little attention to issues regarding Humanities participation in the research agenda; rather, the Human Sciences are left in the background of proposals which are then unclear as to how the latter area will be brought in over the course of the next decade. Yet it is a commitment which must be taken seriously. Our purpose here is to raise the latest questions posed from within the field of Humanities, calling for the inclusion of these specific, urgent and socially relevant issues in the Decadal Survey Astrobiology 2023-2032. Matters linked to studies produced within Humanities and Social Sciences remain on the margins or at the edges of the official speeches and documents that are supposed to demonstrate Astrobiology's commitment to these areas, as in the Appendices of the Astrobiology Strategy (2015) in which they are relegated to the document's final pages.

We emphasize here that, when it comes to scientific research on outer space, the act of reducing the importance of the Humanities is a dangerous setback. Space research depends on public funding and policies, and, due largely to poor communication, the average citizen tends to be suspicious of this type of investment. Efficient strategies should be pursued if such problems are to be overcome. Anthropology, Psychology, Philosophy and Sociology are disciplines have studied how humankind has evolved through the development of short-term skills, such as collecting food or identifying allies and enemies, and it is arguable that this ability to make choices also includes survival strategies for our Defense and Planetary Protection. There are many reasons that people find it difficult to recognize the strong link between outer space investigations and their improved quality of life, benefits that are related to such matters as the clothes we wear, current cancer therapies, and more. Bearing in mind that space research is a long-term undertaking that involves many complex and counter-intuitive scientific issues, it is not surprising that the public entertains so many misconceptions about topics such as "the colonization of Mars", "seeking extraterrestrial evidence for life”, "exoplanetary research”, “Alien Ocean” and other related questions.

This year, the Astrobiology Committee meetings during Space Science Week reported on summaries, data and projections of future missions, yet without offering a 
dialogue with global society nor plans to include social scientists within a solid project for scientific dissemination and public education over for the next few years. Unfortunately, the Space Science Week of the "The Society for Social and Conceptual Issues In Astrobiology" that was to be held this year at the University of Mississippi had to be canceled, due to the Covid-19 pandemic. We were nonetheless provided with notes that have been made available for future discussion, as well as the Space Science Context, a response to the latest research meetings involving Astrobiology and announced in the Boundaries of the MIT Science and Technology Studies Northeast Graduate Student Conference in 2019. We propose that these community efforts include the establishment of focused research coordination networks on the development of human relations on life beyond Earth. The discussion we provide here points to the relevance of including, in upcoming years, activities aimed at building the community of researchers concerned with the social implications of Astrobiology, stimulating interdisciplinarity, collaboration and research on priority issues and actively communicating findings to different audiences and broader society. When disseminating scientific discoveries and their relationship with Astrobiology to different audiences, it is important to recognize areas of agreement and disagreement, taking their ethical, legal, psychological, historical, educational, anthropological, sociological and cultural implications into account.

\section{The social implications of Astrobiology for the next decade}

We recognize the existence of a broad societal interest in society that encourages scholars in the social sciences and humanities to develop and expand public relations through a language that is accessible to all cultures and peoples, with an emphasis on public education and scientific dissemination. Although official documents express concern over Planetary Protection and Defense, contamination and other factors over which humankind maintains direct impact, such mentions have not been enough to remedy the existing gap in scientific production or the disparities between Natural Sciences and the Humanities in relation to the field of Astrobiology. The multiple questions that have arisen and been shared by scientists themselves over the course of the years have been raised over the whole range of the Humanities and deserve an explicit space for discussion in plans for the next decade. The Humanities cannot be excluded from participation in scientific discoveries that could define the future of life here on Earth and beyond. Below we list some of fundamental questions and considerations that emerge from social perspectives.

\section{Recurrent considerations for development over the next decade:}

a. Information sharing and activation of collaboration between humanities researchers across the globe.

b. Creation of a vocabulary of Astrobiological Science that is comprehensible to the general public, as an active contribution to public education and scientific dissemination.

c. Publication of a Dictionary of Astrobiology, following current examples (Dictionary of Astronomy, Dictionary of Chemistry, etc.) and in a user-friendly language.

d. The need for Astrobiology to act against the scientific isolation caused by the technological advancement and which tends to estrange peoples and cultures that are not experiencing high space- technology demand. 
e. Providing an alert regarding the centralization of information from only one scientific field which does not include Human Sciences in its debates, as well as the urgent need to include

scientific contributions that emerge from a diverse variety of cultural narratives.

f. In the absence of moral and ethical responsibilities (which at present, remain undefined), it is necessary to consider the social impact of approaches to, and definitions and interpretations of the origin and evolution of life in the universe, on decisions applied in the legal, political and - above all - private sector spheres.

g. Contemporary philosophy reaffirms that science fiction must be taken seriously as an instrument to discuss and disseminate scientific information and ethical reflections. Screenwriters and authors must be considered important allies with regard to the dissemination of scientific findings.

\section{Recurrent questions to develop over the course of the next decade:}

1). How transparent are information and discoveries for the promotion of knowledge about intelligence and life beyond Earth? What is Astrobiology's contribution to the history of the development of human thought and the social relations that circumscribe it?

2). Do all missions involve universal guidelines and protocols for planetary protection? What do they consist of?

3). What impact may our society have on other possible worlds? What are the moral and ethical implications of expanding human life beyond Earth?

6). How can lunar, oceanic, and Mars missions be explained to the general public in an educational and democratic way that stimulates the interest and engagement of society?

7). How can information be shared, on the basis of the scientific dissemination between research centers in Astrobiology, public education, and society as a whole, throughout the world?

8). In a society that is overloaded with information - which is not always correct, nor adequately conveyed - what strategies can be developed to allow for greater dissemination of real scientific information?

\section{Astrobiology Networks and the Humanities}

In its final chapter and appendices, the Astrobiology Strategy that was launched in 2015 outlined an interest in bringing the Human Sciences closer to Astrobiological studies over the course of the next decade. Although these challenges and opportunities remain distant from what scientists and researchers from other networks in Astrobiology address, the recurrent topics of congresses, meetings, workshops, webinars, and publications must be considered. These distinct and diverse networks of astrobiologists are interested in scientific dissemination, in the inclusion of more researchers and the public, and, above all, in debate within a civil society that yearns for 'a seat at the table'. It is necessary that standards and practices point out the limits of our current language on Planetary Protection and Defense, as both space and Astrobiology belong to everyone. It is stimulating and challenging to encounter solid networks of astrobiologists engaged in the Human Sciences and committed to reinforcing the Principles of Astrobiology, thereby expanding their social mission. To name a few of these principles: 
- NASA and other space agencies have an obligation to comply with the Planetary Protection policy and engage society in developing new regulations that take social demands into account, as well as in facing the challenges and potential conflicts of interest between Astrobiology and commercial use of outer space.

- The creation of an astrobiological vocabulary of easy public use that is able to encourage the engagement of both scientific dissemination and public education. Clear, consistent and effective international protocols are needed to guide a process to assess such impacts, from the perspective of different cultural narratives, coming from the academic community, independent researchers and the public.

- The astrobiological community must take scientific communication seriously, given existing risks of estrangement from society. Interdisciplinary strategies are rarely available to the public and when present, clearly reveal that the Humanities have been neglected in the context of important events.

The community of researchers in the Humanities who are interested in Astrobiology has demonstrated active and growing participation in Global Congresses. Space Science Week, which was held remotely due to the global Covid-19 pandemic, raised alerts for the Humanities area. Yet there was no increased willingness to stake out a Committee position on greater inclusion of the Humanities within NASA's Department of Astrobiology. Future missions and their specialties were addressed with no mention of the considerable contribution that researchers in Human Sciences and Astrobiology are uniquely capable of making regarding the political, social, ethical aspects of Planetary Protection and Defense. In this context, the discrete absence of an astrobiological debate that engages with society is serious; its official implementation for the next decade is urgent.

\section{Astrobiological Group of Social Issues for the Decadal Survey Astrobiology 2023- 2032}

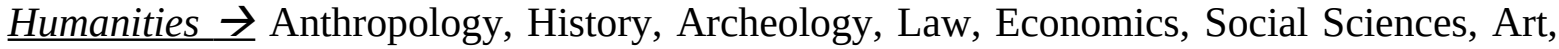
Literature, Philosophy, Psychology, Communication, Design, Photography, Politics, Journalism, etc.

Based on questions asked by researchers from the realm of the natural sciences who seek to address phenomena ranging from the origin of life to the biological possibility of human inhabitation of other worlds, Astrobiology has largely neglected to incorporate the Humanities into its corpus of narratives and discourse. By sharing our symbolic imagery and revisiting universal archetypes, Astrobiology may be able to develop its sensitivity to the historical and political situations that characterize post-colonial scientific processes. Below, we present some of the contemporary issues that Human Sciences researchers linked to the astrobiological field have brought out:

- Research on human beings' relations to life and extreme environments on Earth.

- Possible relationships between humans and other viable worlds. How can we consider the collective future of Humanity in outer space? The colonization process of outer space exploration has a social context that must be examined.

- What possibilities exist for human governance beyond Earth?

- With regard to contemporary possibilities such as asteroid mining by private companies, is the Outer Space Treaty sufficient or ambiguous? Should it be changed? 
- Examination of the ethical, legal, moral, and cultural conducts that result from scientific research on Astrobiology and its delegations. Changes in individuals, cultures and interpretations of the origin of life through the contributions of Astrobiology.

- Definition of Planetary Protection and Defense for peaceful purposes, its policies, and protocols.

- The need to understand the real natural and technological threats to the continuation of life on Earth as gleaned from experiments in outer space; deliberations on a plan for outer space based on its social effects and implications for the entire terrestrial population.

- International cooperation and participation in making decisions that have an impact on life on Earth.

- Risk factors for a technological civilization include global catastrophes such as nuclear winter, pandemic, asteroid impacts, and future technological failures of artificial intelligence, which must be reported to society immediately. The dialogue between the Astrobiological field and society must always be edifying for humanity.

\section{$\rightarrow$ Group actions for the next decade}

a. Further development of an interdisciplinary basis for work among social scientists in Astrobiology. More convening of lectures and posters from the human sciences in Astrobiology events.

b. Elaboration of a database for astrobiology researchers focusing on issues related to the Humanities. Encouraging grants and fellowships for humanities researchers who want to address pressing topics in Astrobiology.

c. Encouraging virtual meetings and prioritize the social implications that Astrobiology holds for different spheres of social life.

d. Relating cultural narratives to ongoing research and encourage the circulation of information amongst the widest possible audience, through scientific dissemination and public education.

e. Examining the deficiencies in social arenas that are not being properly incorporated or engaged and attempt to fill in these gaps. Turning Astrobiology into a community-oriented science within the Social Sciences today.

f. Including global contact with children and encourage children's curiosity on astrobiological issues and mysteries. Creating a more inclusive global community around issues of astrobiology and outer space, including inclusive 'outer space' cultures for the disabled.

g. Promoting deep debate on issues of race and gender (racism, misogyny and colonial roots) and a possible future in outer space. Reassessment of the place that has been awarded to racial and ethnic minorities in planetary science.

\section{Final considerations}

Interdisciplinary perspectives are as fundamental as any others within the history of Astrobiology and must, urgently, be stimulated, developed, and disseminated. The Astrobiological Group of Social Issues for the Planetary Science and Astrobiology Decadal Survey 2023-2032 is intended as a vehicle for increasing the communication and collaboration of Humanities scientists within Astrobiology and in terms of its historical composition as an interdisciplinary and multidisciplinary science, recovering its 
foundational principles foundation and committing to follow them for years to come. The result of the emergence of this interdisciplinary movement in recent years has been to initiate a quest for better and more informed decision-making on Astrobiology actions, and one that is able to engage and involve everyone on Earth. This community of researchers is interested in discussing the issue of the potential impact of Astrobiology on society. Finally, we must build potential studies in the area of the Humanities for the benefit of future generations, examining the stages of social progress that are needed if astrobiological perspective are to be fully understood. If this does not occur, the scientific community will face the serious risk of separating itself from crucial political discussions, increasingly dangerous not only for the continuity of efforts oriented toward outer space, but for our daily life here on Earth. Interdisciplinary and multidisciplinary progress and expansion in Astrobiology have grown within the scientific field that engages with life, relationships, and social and outer space communities.

Social Sciences and Humanities concentrate in this case on the importance of involving multiple disciplines to understand and characterize diverse views of life, relationships, and natural environments and how they have evolved over time. Expanded exploration of outer space raises ethical questions about human experimentation, legal responsibilities, risks, and moral imperatives in relation to life. The prospect of long-term changes in humans enters the new conceptual territory, particularly with the possible engineering of new humans, non-humans, or hybrid beings.

Communication on this has been largely limited to the astrobiology audience. Social science experts do not routinely interact with the astrobiology research community and are often unaware of its complex issues. In addition, communication and engagement with the public on non-scientific topics is generally not included within scientific dissemination of astrobiology. In conclusion, interdisciplinary perspectives are equally important parts of the unfolding history of astrobiology and must be communicated to the public and space communities. The Planetary Science and Astrobiology Decadal Survey 2023-2032 for the next decade could serve as a vehicle to increase communications and collaboration between scientists and non-scientists - and to proactively help expand communication on ideas articulated through the Principles of Astrobiology. It is our hope that, over time, the result of this interdisciplinary research will lead to better and more informed decision-making and actions for all. These efforts are to gradually build a community of interdisciplinary researchers interested in examining questions regarding the impact of astrobiology on society, as well as the potential impact of society on astrobiology.

\section{References}

National Research Council 2003. New Frontiers in Solar System Exploration. Washington, DC: The National Academies Press $\rightarrow$ https://doi.org/10.17226/10898

National Research Council 2011. Vision and Voyages for Planetary Science in the Decade 2013-2022. Washington, DC: The National Academies Press. $\rightarrow$ https://doi.org/10.17226/13117.

National Academies of Sciences, Engineering, and Medicine. 2017. Report SeriesCommittee on Astrobiology and Planetary Science: Getting Ready for the Next Planetary Sciences Decadal Survey. Washington, DC: The National Academies Press. $\rightarrow$ https://doi.org/10.17226/24843 
National Academies of Sciences, Engineering, and Medicine 2017. Powering Science:

NASA's Large Strategic Science Missions. Washington, DC: The National Academies Press. $\rightarrow$ https://doi.org/10.17226/24857.

National Academies of Sciences, Engineering, and Medicine 2018. Visions into Voyages for Planetary Science in the Decade 2013-2022: A Midterm Review. Washington, DC: The National Academies Press. $\rightarrow$ https://doi.org/10.17226/25186.

National Academies of Sciences, Engineering, and Medicine 2019. Report Series: Committee on Astrobiology and Planetary Science: Review of the Planetary Science Aspects of NASA SMD's Lunar Science and Exploration Initiative. Washington, DC: The National Academies Press. $\rightarrow$ https://doi.org/10.17226/25373.

National Academies of Sciences, Engineering, and Medicine 2019. Report Series: Committee on Astrobiology and Planetary Science: Review of the Commercial Aspects of NASA SMD's Lunar Science and Exploration Initiative. Washington, DC: The National Academies Press. $\rightarrow$ https://doi.org/10.17226/25374.

National Research Council 2008. Grading NASA's Solar System Exploration Program: A Midterm Report. Washington, DC: The National Academies Press. $\rightarrow$ https://doi.org/10.17226/12070.

Astrobiology and Society: Building an Interdisciplinary Research Community $\rightarrow$

Astrobiology Strategy. National Aeronautics and Space Administration, 2017. $\rightarrow$ https://nai.nasa.gov/media/medialibrary/2015/10/NASA Astrobiology Strategy 20151510 08.pdf

Space Science in Context, a virtual conference. $\rightarrow$

https://spacescienceincontext.wordpress.com/, 14th, May, 2020. 